\title{
Hypothesis: Regulation of neuroplasticity may involve I-motif and G- quadruplex DNA formation modulated by epigenetic mechanisms
}

\author{
German Todorov, Catarina Cunha* \\ Emotional Brain Institute, Nathan Kline Institute, Orangeburg, NY, USA \\ ${ }^{*}$ Correspondence: Catarina Cunha, Emotional Brain Institute, Nathan Kline Institute, Orangeburg, NY, \\ USA, E-mail: catarina.cunha@nki.rfmh.org
}

\begin{abstract}
Recent studies demonstrated the existence in vivo of various functional DNA structures that differ from the double helix. The G-quadruplex (G4) and intercalated motif (I-motif or IM) DNA structures are formed as knots where, correspondingly, guanines or cytosines on the same strand of DNA bind to each other. There are grounds to believe that G4 and IM sequences play a significant role in regulating gene expression considering their tendency to be found in or near regulatory sites (such as promoters, enhancers, and telomeres) as well as the correlation between the prevalence of G4 or IM conformations and specific phases of cell cycle. Notably, G4 and IM capable sequences tend to be found on the opposite strands of the same DNA site with at most one of the two structures formed at any given time. The recent evidence that $\mathrm{K}^{+}$, $\mathrm{Mg}^{2+}$ concentrations directly affect IM formation (and likely G4 formation indirectly) lead us to believe that these structures may play a major role in synaptic plasticity of neurons, and, therefore, in a variety of central nervous system (CNS) functions including memory, learning, habitual behaviors, pain perception and others. Furthermore, epigenetic mechanisms, which have an important role in synaptic plasticity and memory formation, were also shown to influence formation and stability of G4s and IMs. Our hypothesis is that non-canonical DNA and RNA structures could be an integral part of neuroplasticity control via gene expression regulation at the level of transcription, translation and splicing. We propose that the regulatory activity of DNA IM and G4 structures is modulated by DNA methylation/demethylation of the IM and/or G4 sequences, which facilitates the switch between canonical and non-canonical conformation. Other neuronal mechanisms interacting with the formation and regulatory activity of non-canonical DNA and RNA structures, particularly G4, IM and triplexes, may involve microRNAs as well as ion and proton fluxes. We are proposing experiments in acute brain slices and in vivo to test our hypothesis. The proposed studies would provide new insights into fundamental neuronal mechanisms in health and disease and potentially open new avenues for treating mental health disorders.
\end{abstract}




\section{I-motif and G-quadruplex}

While double helix DNA structure (B-form) is the most common conformation, a variety of other forms are known to exist in vitro ${ }^{1-5}$. Some forms also appear to exist in vivo and were shown to have physiological significance ${ }^{6}$. Of those, G-quadruplex (G4) and intercalated motif (I-motif or IM) appear to be the most interesting. G4 is found in G-rich regulatory regions of the genome and was demonstrated to exist in vivo in several studies ${ }^{6,7}$. There is also evidence of its involvement in regulatory pathways ${ }^{8}$.

$\mathrm{IM}$, a form occurring primarily in C-rich regulatory regions and are characterized by $\mathrm{C}: \mathrm{C}+$ pairs. Hence IMs form more easily at lower $\mathrm{pH}$. However, IMs can form at normal $\mathrm{pH}$ and are also affected by other factors, including ionic strengths $\left(\mathrm{K}^{+}, \mathrm{Mg}^{2+}\right.$ concentration in particular), sequence, DNA anti-helicity torsional strain, and molecular crowding. A recent study provides a strong indication that IM too exists in vivo and has a physiological role' ${ }^{1}$.

Many IMs occur in strands complimentary to G4 sequences and vice versa. There is some evidence of cases when G4 and IM can form simultaneously in the opposing strands, but there appears to be more evidence that one forms to the exclusion of the other (in the opposite strand), which may represent a bi-directional regulatory mechanism ${ }^{2,5}$.

\section{Cell cycle}

The levels of both IM and G4 appear to change depending on the phase of cell cycle, which appears correlated with changes in gene activity / transcription patterns. A minimum of IM was reported to occur at G0/G1 boundary and the maximum at G1/S boundary ${ }^{6}$. On the other hand, G4 formation appears to occur predominantly in S phase ${ }^{9}$.

IM has been tentatively linked with promoters of several proto-oncogenes (such as c-myc, Bcl2 ), which play a role in cell cycle and apoptosis ${ }^{10}$. This appears to be consistent with its higher occurrence at G1/S boundary when many proto-oncogenes are active ${ }^{11}$.

\section{G4 and methylation}

Methylation appears to affect the formation and stability of G4 and thereby modulate the effect of G4-capable sequences on transcription regulation ${ }^{12,13}$. Tsukakoshi and colleagues found that 
the binding of the transcription factor SP1 to the G4-forming DNA sequence in the promoter of VEGF gene can be altered by CpG methylation. They showed the methylation state of the G4 sequence to correlate with changes in G4 structure/topology and VEGF expression, indicating the role of G4 conformation and its methylation state in transcription regulation.

In another study, CpG methylation was also shown to affect thermostability of G4 in the VEGF promoter $^{8}$. This effect was dependent of the specific CpG position within G4 sequence as well as the presence of $\mathrm{K}^{+}, \mathrm{Na}^{+}, \mathrm{Mg}^{2+}$ ions. Also, $\mathrm{CpG}$ methylation in G4-forming sequence of MEST gene promotor was shown to interfere with polymerase chain reaction by stabilizing the G4 formation and thus causing the polymerase arrest ${ }^{14}$.

Furthermore, using G4-ChIP-sequencing of human genome, Shi-Qing Mao and colleagues found that most G4 structures are formed in regions containing unmethylated $\mathrm{CpG}$ islands (CGIs). Additionally, they showed co-localization of G4s and DNMT1 binding at CGls, in which G4s restrain DNMT1-mediated methylation. Mao and colleagues proposed that G4s' role involves conserving gene expression function of the corresponding sites by shielding them from methylation through isolating and constraining DNMT1 activity ${ }^{15}$.

\section{I-motif and methylation}

Fairly little is known about the role of methylation in formation, stability and physiological role of IMs. In-vitro evidence indicates that cytosine methylation at position 5 increases the $\mathrm{pH}$ of midtransition to IM form (by +0.11 ) whereas hydroxy-methylation reduces it (by -0.2$)$. If this effect holds in vivo, then methylation may facilitate IM conformation in vivo while hydroxy-methylation could have the opposite effect ${ }^{16}$.

Another in vitro study (using oligonucleotides) reported that CpG methylation of IMs altered the energetic barrier of dsDNA <-> IM transition. For some IM sequences, methylation had stabilizing effect, which was reduced or reversed by molecular overcrowding ${ }^{17}$.

\section{Hypothesis: IMs and G4 may play a regulatory role in neuroplasticity}

Synaptic plasticity represents a mechanism in neurons whereby information input is turned into long-lasting changes in neuronal connectivity essential to memory, learning, cognition and 
adaptive behavior. The underlying mechanisms include a variety of activity-dependent molecular systems, such as histone modification, DNA methylation and nucleosome remodeling, which in turn alter and coordinate gene expression involved in long-term potentiation (LTP), long-term depression (LTD), and synaptic scaling, the key physiological "algorithms" involved in memory formation and other neural functions ${ }^{18-21}$.

There is emerging evidence that IMs occur in vivo and their formation varies depending on the phase of cells cycle. Also, putative IM patterns are found in many of the genes involves the regulation of cell cycle and apoptosis (see Cell Cycle section above).

G4 have been well demonstrated to occur in vivo, appear to have some role in transcription regulation and their formation also varies depending on the phase of cell cycle (see Cell Cycle section above).

Also, IM and G4 patterns often occur together in the opposite strands but tend to be mutually exclusive conformations (in most cases), i.e. either one or the other form occurs at any given time. This appears to provide a molecular mechanism for a bi-directional regulatory switch controlling a potentially large set of genes whose regulatory sequences, such as promoters and enhancers, include IM/G4 patterns. Such mechanism could have a major role in a cocoordinated, potentially bi-directional regulation of the substantial number of genes involved in progression through phases of cell cycle ${ }^{2,5}$.

While cell division and hence cell cycle regulation is of central importance for many tissues and cell types, terminally differentiated neurons generally do not divide. In adult brain, de novo neurogenesis occurs at fairly low rates (if at all - still some controversy) and may be limited only to certain areas (such as hippocampus) ${ }^{22}$.

As opposed to other cell types, adult neurons adapt to changing conditions not via cell division or death (apoptosis) but by forming new neuronal connections (neurite growth/new synapse formation) and/or eliminating existing ones (synaptic pruning) or LTP and LTD, correspondingly ${ }^{18-21}$.

Notably, many of the genes (including several proto oncogenes) involved in regulating cell cycle and apoptosis also play a role in LTP and LTD. In a sense, this is not surprising: while neurite growth, as opposed to cell growth, does not require nuclear DNA replication and cell division, both require an increase in energy production, protein synthesis, biogenesis of ribosomes and 
mitochondria, etc ${ }^{23}$. Conversely, both apoptosis and synaptic pruning require activation of autophagy, inhibition of energy production, protein synthesis and so forth ${ }^{24} 23$.

There appear to be many similarities in the regulatory pathways and mechanisms involved in cell division/apoptosis and LTP/LTD. In the light of the emerging evidence of the role of IM and G4 in regulating the genes involved in the control and progression of cell cycle and apoptosis, we propose that IM and G4 also have a regulatory role in neuroplasticity, including LTP, LTD, and synaptic scaling. In particular, one of the well-researched $\mathrm{IM}$ sequences is in Bcl-2 promoter $^{25,26}$. Bcl-2 is known to be involved in apoptosis and autophagy, partly by inhibition of mTOR pathway ${ }^{27}$. Notably, mTOR pathway was shown to be involved in various aspects of neuroplasticity, including LTP, LTD and memory consolidation ${ }^{28-30}$. This putative new mechanism may not only improve our understanding of neuroplasticity regulation but also open a new direction in drug development for neurological and mental health conditions (see Clinical implications).

There is evidence that some neurodegenerative diseases may be caused by aberrant G4 formation interfering with transcription. The most common genetic cause of amyotrophic lateral sclerosis (ALS) and frontotemporal dementia (FTD) is the hexanucleotide repeat expansion (HRE), (GGGGCC)n, in C9orf72 gene. Haeusler and colleagues showed that the molecular mechanism of that pathology is based on HRE forming DNA and RNA G4s with distinct structures giving rise to aberrant variants of RNA DNA hybrids (R-loops) ${ }^{31}$. The structural polymorphism in HRE causes a repeat-length-dependent accumulation of aberrant transcripts aborted in the HRE region. The transcribed HRE repeats were shown to bind to ribonucleoproteins (specifically nucleolin) in a conformation-dependent manner contributing to nucleolar stress and initiating cascades leading to ALS/FTD.

Tyrosine hydroxylase is the enzyme involved in the limiting step of catecholamine biosynthesis, the conversion of tyrosine to DOPA, which has been shown to play important roles in the nervous system functions, including learning, memory, synaptic plasticity, cognition, reward, motor function and others. Tyrosine hydroxylase mutations and variations have been associated with several neurological and psychiatric disorders including Parkinson's disease and Schizophrenia ${ }^{32,33}$. The regulatory regions of tyrosine hydroxylase gene appear to include and be regulated by $\mathrm{G} 4$ and I-motif structures ${ }^{34,35}$. 
Hypothesis: The regulatory effects of DNA methylation on neuroplasticity may, in part, occur via methylation-induced changes in the stability/formation of IM and G4

There is growing evidence that epigenetic mechanisms play a major role in neuroplasticity ${ }^{36}$. In particular, DNA methylation/demethylation appears to be especially important in inducing and maintaining long-lived circuitry changes, such as those associated with long-term memory, learning, habits, addiction, fear extinction and so forth ${ }^{36-39}$.

If IM and G4 indeed play a role in neuroplasticity regulation, then methylation/demethylation of DNA in IM/G4 sequences could modulate the regulatory signals by facilitating or hindering the formation on these non-canonical conformations.

This may occur in one of the following ways:

1) Methylation state may alter the stability of IM and/or G4 directly

Methylation, hydroxy-methylation or de-methylation of IM may alter its stability, increasing/decreasing its prevalence in vivo. For example, based on in vitro data mentioned above, methylation of IM pattern (via DNMT enzymes) may increase IM formation whereas hydroxy-methylation of methylated cytosine (via TET enzymes) would have the opposite effect. Similar methylation-related effects may be expected for G4. For example, CpG methylation appears to affect G4 stability and transcription factor binding to VEGF promoter ${ }^{12}$.

2) Methylation state may affect formation of IM or G4 in one strand, thereby preventing the formation of the regulatory (transcription-factor binding) G4 or IM, correspondingly, in the opposite strand.

This is based on findings that IM and G4 patters often co-occur in opposing strands and the formation of one appears to usually suppress the other and vice versa. Therefore, if IM (or G4) sequence in a sense strand has a direct regulatory role (e.g. binds a transcription factor), then methylation of G4 sequence (or IM, correspondingly) in anti-sense strand, may have indirect regulatory affect by suppressing the IM (or G4) conformation in the sense strand.

3) $\underline{I M}$ and/or G4 may impact neuroplasticity by affecting MeCP2 function

MeCP2 is a regulatory factor that has a role in neuroplasticity. MeCP2 binds to the common DNA methylation site CpG, which changes the expression of multiple genes. MeCP2 has essential role in early CNS development as well as maintenance role in mature adult neurons 
where it modulates both synaptic development and function. The ability of MeCP2 to bind CpG site may be affected when the $\mathrm{CpG}$ is within or close to IM or G4 conformation.

A less direct but intriguing possibility is that MeCP2 transcription, splicing or translation may be affected by IM and/or G4 formation. Bagga and colleagues showed that Rett syndrome mutations resulting in the decreased MeCP2 protein expression was associated with a conserved G4. They also identified G4s that could regulate MeCP2 pre-mRNA alternative splicing in either negative or positive way ${ }^{40}$.

4) IM may be involved in learning and memory regulation and affected by non-CpG (particularly $\mathrm{CpC}$ ) methylation via DNMT3A

While the majority of methylated cytosines are found at $\mathrm{CpG}$ sites, non-CpG methylations ( $\mathrm{CpA}$, $\mathrm{CpT}$ and $\mathrm{CpC}$ ) also occur and can induce gene silencing or activation depending on the region. However, non-CpG methylation appears to be restricted to a few specific cell types, notably neurons and glial cells. Non-CpG methylation in mammals is performed by DNMT3A and DNMT3B members of DNA methyltransferase enzyme family ${ }^{41}$.

In terminally differentiated neurons in adult brains, DNMT1, DNMT3A and DNMT3B appear to be active. DNMT3A appears to be the specific subtype required for normal de novo learning and memory. Forebrain specific DNMT3A knockout mice, but not DNMT1 knockout mice, had altered LTP and synaptic plasticity in the affected areas and showed learning and memory deficits ${ }^{42}$.

IM is a C-rich pattern that usually includes repeating $C[C]_{n}$ clusters providing a potential target for $\mathrm{CpC}$ methylation specifically by DNMT3A (or DNMT3B). Considering the role of DNMT3A in learning, memory and likely other related instances of LTP, we propose that DNMT3A may act, in part, by methylating cytosines inside IM patterns, facilitating IM formation and thereby modulating expression.

It is notable that whereas non-CpG methylation is higher in the brain than in most other tissues (where it is minimal), different neuron types vary by level and distribution of both $\mathrm{CpG}$ and nonCpG methylation, possibly reflecting functional differences ${ }^{43}$. It is unclear whether and which TET enzymes have any specificity in regard to demethylating CpG vs non-CpG sites, DNA sequence or conformation, such as IM or G4. Investigating this possibility appears worthwhile. 
Hypothesis: The regulatory effects of DNA methylation on neuroplasticity may by modulated by IM and G4, whose formation may interfere with epigenetic signaling pathways, such as methylation/demethylation and histone acetylation/deacetylation activity.

Above we hypothesized that DNA methylation may affect the formation of IM and G4 and thus interfere with signal transduction and gene expression involved in neuroplasticity. We believe that the opposite may also take place, i.e. the formation of G4 or IM may modulate the activity of epigenetic mechanisms, making the connection between G4/IM and epigenetic modifications somewhat bi-directional. There is emerging evidence pointing to such possibility. In a recent study, Mao and colleagues found that G4 can bind to and thus inhibit the activity of DNMT1 enzyme leading to reduced level of DNA methylation, particularly at and near the binding site ${ }^{15}$. If so, the factors inducing G4 formation would presumably reduce DNA methylation levels, potentially altering gene expression. In most, albeit not all cases, DNA demethylation upregulates the expression of directly affected genes. Furthermore, considering that IM and G4 tend occur in the opposing strands of the same sequence and their formation tends to be mutually exclusive, IM may have the opposite effect to G4 on DNA methylation of the corresponding sites. Hence, the factors that induce IM formation would tend the suppress G4 formation in the opposing strand and reduce the inhibitory binding for G4 to DNMT1, leading to greater DNMT1 activity and increased DNA methylation.

A recent study by Shioda and colleagues indicates that such effects not only occur in vivo but are relevant to the neuronal functions and potentially to neuroplasticity ${ }^{44}$. The researchers demonstrated that ATRX, a chromatin remodeling protein, acts by binding to $\mathrm{G} 4$ of the imprinted XIr3b gene, regulating its expression. In turn, XIr3b binds to dendritic mRNAs and its overexpression impairs dendritic transport leading to synaptic dysfunction. ATRX gene mutations cause alpha-thalassemia $X$-linked intellectual disability (ATR-X) syndrome. Notably, ATRX was shown to bind to G4s in XIr3b together with DNMTs. Furthermore, the levels of DNMT1 and DNMT3A, the DNMT subtypes involved in adult neuroplasticity, were decreased (relative to wild type) in ATRX-deficient mutant mice in G4-rich regulatory site for XIr3b.

While there is already emerging evidence that G4s and indirectly IMs can affect DNMT enzymes and thus alter local methylation levels, it is conceivable that other enzymes involved in epigenetic modifications may bind to and be inhibited/activated by G4s or IMs. It appears 
worthwhile to research the effects of G4s and IMs on the activity of ten-eleven translocation methyl cytosine dioxygenases (TET family), histone deacetylases, histone acetyltransferases, histone methyltransferases and other enzymes and factors involved in epigenetic modifications occurring during neuroplasticity.

All in all, the relationship between G4, IM and epigenetic modifications in the context of neuroplasticity is likely to exists, be complex and vary depending on neuron subtype, external signals, phase of cell cycle and other variables. This is consistent with the recent findings that epigenome in neurons is not fixed but is in a state of equilibrium dynamically maintained by multiple mechanisms and shifted in different directions depending of a particular type of neuroplasticity, such as during LTP, LTD and synaptic scaling.

\section{Hypothesis: The regulatory activity of microRNAs in neuroplasticity and elsewhere may be modulated by their effects on the formation and stability of DNA G4s and IMs. G4 formation in microRNA transcripts may also have a regulatory role.}

Micro RNAs (miRNAs) are known to have multiple regulatory roles in all tissues, including the brain. Abnormal levels of various miRNAs were found in many neurological and mental health conditions ${ }^{45}$. There is evidence that DNA G4 conformation may hinder the DNA binding activity of relevant miRNAs thus affecting their regulatory function ${ }^{46}$. Similar effect of miRNA on DNA IM formation appears possible as well. Hence cellular events that affect the transition of DNA to/from IM and/or G4 conformation could affect signaling mediated by some miRNAs.

Also, G4 in miRNA transcripts were shown to affect their processing and maturation, which may modulate regulatory function of miRNAs ${ }^{47,48}$.

\section{Hypothesis: RNA G4 may play a key role in neuroplasticity through translation regulation and splicing control.}

Translation regulation plays a major role in synaptic plasticity, memory formation and related circuitry changes and occurs via multiple mechanisms ${ }^{49-51}$. Notably, dendrites contain a large amount of locally pre-positioned ribosomes and mRNA ready for rapid onset on translation occurring during LTP ${ }^{52-54}$. We propose that RNA G4s may play important role in regulating translation during neuroplasticity. 
There is considerable evidence that G4s can form in RNA and may be more stable than their DNA counterparts in vitro ${ }^{55,56}$. There is also evidence that RNA G4s play a role in translation regulation by, for example, interfering with initiation or causing early termination of translation 57,58. RNA G4s also may be involved in the regulation of alternative mRNA splicing ${ }^{59}$. The genomes of eukaryotes, including humans, appear to have a large number of transcribed sequences of putative RNA G4s ${ }^{60}$. However, Guo and Bartel have recently reported that the majority of G4 regions in eukaryotes in vivo exist in the unfolded state at any given time ${ }^{56}$. Notably, the model RNA G4 regions unfolded in eukaryotes became folded when ectopically expressed in Escherichia Coli. Guo and Bartel suggested that eukaryotes have the machinery that globally unfolds the majority of RNA G4s in order to suppress their ability to interfere with translation, whereas bacteria, lacking such machinery, have undergone evolutionary depletion of G4-forming sequences. The mechanism of active RNA G4 unfolding in eukaryotes remains to be determined and appear to involve RNA-binding proteins, such as hnRNP family ${ }^{61,62}$ and certain helicases (the enzymes unwinding DNA and RNA), such as Pif1 ${ }^{63}$.

In the light of the above, it appears that, on one hand RNA G4-forming sequences are common in eukaryotes, are thermodynamically stable and can easily form spontaneously while, on the other hand, their formation is actively controlled and at least partly suppressed in most cases most of the time. We propose that such situation produces a metastable, actively maintained equilibrium suitable for a fine control of translation (and perhaps also splicing), which is crucial for the regulation of neuroplasticity. For example, an external signal or a miRNA could reduce the expression or impair the binding ability of an G-rich-RNA-binding protein, leading to increased formation of G4s, reduced translation in dendrites and eventually LTD. A similar mechanism that increases the unfolding of certain G4s may lead to LTP. The potentially significant role of RNA G4s in neuronal function and neuroplasticity appears to be supported by the evidence associating transcribed G4-forming sequences with a number of mental health disorders ${ }^{64}$.

Fragile $X$ syndrome (FXS) is the most-frequent heritable syndrome of mental insufficiency. It is caused by the expansion of (CGG)n repeats in the FMR1 gene leading to the loss of the corresponding protein FMRP. FMRP is involved in regulating the translation of specific neuronal messenger RNA (mRNA) targets and contains an arginine-glycine-glycine (RGG) RNA-binding domain shown to bind with G4 forming mRNA structures ${ }^{65}$. RGG motif of FMRP also modulates its association with polysomes indication the role in translation regulation ${ }^{66}$. 
Additionally, MAP1B, PP2A and Shank1 genes that are essential for neural development have been shown to harbor one or more G4s in their 50-UTRs and/or 30-UTRs and their expression was shown to be upregulated by the absence of FMRP, indicating that FMRP plays pivotal a role in neonatal brain development ${ }^{67,68}$.

\section{Hypothesis: Neuronal ion and proton fluxes may produce some of their regulatory/signaling by affecting the formation and stability of RNA G4, IM, and triplex structures}

The formation of many non-canonical nucleic acid forms, such as IM, G4 and triplexes is affected by $\mathrm{pH}$ and ion concentrations, particularly potassium and magnesium 69-74. Physiological variations in these parameters may exert some of their regulatory effects by facilitating or inhibiting the formation of these non-canonical structures.

Ion fluxes, including fluxes of potassium and magnesium ions, have a particularly large role in neurons. The greatest changes in neuronal ion concentration presumably occur in the cytosol, where they may affect RNA (and perhaps mit-DNA) conformation ${ }^{75}$. RNA G4 formation under physiological conditions is well established and there is considerable evidence supporting its regulatory significance (as we discussed above). Furthermore, potassium ions were shown to stabilize RNA G4 whereas magnesium and calcium ions have the opposite effect ${ }^{71}$. Similar effects except in the opposite regulatory direction appear to occur in RNA triplexes, with potassium inhibiting and magnesium facilitating their formation ${ }^{73,74,76}$.

We propose that RNA G4 and RNA triplex conformation changes may be one of the mechanisms by which cytosolic ion fluxes associated with neuronal signaling exert their action. Similar considerations may also apply to $\mathrm{pH}$ changes. Proton fluxes/gradients appear to have a major regulatory role in neurons and glia $^{77}$.

Pre-synaptic activity is known to change extracellular $\mathrm{pH}$ in CNS. Extracellular $\mathrm{pH}$ changes affect neurons via acid-sensing ion channels (ASICs). However, extracellular pH changes may also directly alter intercellular $\mathrm{pH}$ in neurons and glia $^{78}$. Neuronal signaling also appears to be associated with $\mathrm{pH}$ changes in intracellular compartments, such as endosomes ${ }^{79}$ and mitochondria ${ }^{80}$, and potentially with cytosolic proton fluxes ${ }^{81}$. The regulatory role of neuronal proton fluxes may involve, in part, the direct effect of $\mathrm{pH}$ on the stability of cytoplasmic noncanonical nucleic acid structures, such as mitochondrial DNA IMs and RNA triplexes ${ }^{82,83}$. It is 
unclear if $\mathrm{pH}$ or ion concentration changes in the nucleus are sufficient for regulatory effects via DNA IM/G4 but this may be worth investigating.

\section{Clinical implications}

New class/category of drugs for neurological and psychiatric conditions

The conformation and stability of both IM and G4 can be altered by specific chemical agents, which makes them a potential drug target. Some of the relevant chemical agents could be small molecules absorbed via GI tract after oral administration and capable of crossing blood-brain barrier.

For example, a pregnanol derivative and a cholestane derivative have been shown to target IM and affect Bcl-2 expression presumably regulated via IM pattern sequence ${ }^{84,85}$. G4 targeting agents is already a promising area of anti-cancer drug research. However, while designing drugs that target specific G4s is crucial for developing effective treatments, it is a challenge due to the topological similarity of the skeleton of diverse G4s. Nevertheless the next-generation G4

ligands are being developed ${ }^{86,87}$. In a recent study, Shioda and colleagues reported promising results indicating that drugs targeting G4s may have potential as a treatment option. They administered 5-aminolevulinic acid (5-ALA), which reduced the abnormal overexpression of XIr3b gene in the mouse model of alpha-thalassemia X-linked intellectual disability (ATR-X) syndrome. They demonstrated that ATAX suppresses expression of XIr3b by binding to $G 4$ and DNMTs, which does not occur in the ATAX deficient mutant mice. 5-ALA normalized XIr3b expression presumably because its metabolites, protoporphyrin IX and hemin, bind to G4s interfering with the binding of ATAX and DNMTs. This appear to be an early indication of the potential that the drugs targeting IMs and G4s may have in neurological and psychiatric conditions ${ }^{44}$.

\section{Ideas/methodology for testing the hypotheses}

\section{$\underline{\text { Acute brain slices: }}$}

Detecting IM, G4 and triplex formations and observing their dynamics in acute brain slices during/after induced LTP/LTD via specific anti-IM or anti-G4 antibodies in DNA and RNA ${ }^{6,88}$ and fluorescence resonance energy transfer (FRET) ${ }^{89}$. 
Detecting IM and its dynamics in vitro during neuroplasticity LTP/LTD via nuclear magnetic resonance (NMR) spectroscopy ${ }^{86}$. NMR has been used to assign non-exchangeable protons in the nuclear magnetic resonance spectra of small DNA/RNA molecules, in which the groups of protons in specific sugars or bases are first detected by scalar couplings (COSY), and then correlated spatially based on the degree of cross-relaxation due to the Overhauser effect (NOESY). The method appears to be generally applicable to moderate-sized DNA/RNA formations. In cell cultures and brain slices ${ }^{31}$, detection can be used to monitor the levels of intracellular adenosine triphosphate (ATP), other high-energy phosphates and cytosolic $\mathrm{pH}$, whereas ${ }^{3} \mathrm{H}$ detection can be used to monitor lactate and glutamate levels. We hypothesize that this method would be ideal for monitoring the factors affecting IM/G4-formation ( $\mathrm{pH}$ levels, etc) while distinguishing between DNA and RNA forms, such as G4, IM and triplex, simultaneously 90-92.

Homology/pattern search for putative IM and G4 in genes (including regulatory regions) involved in neuroplasticity.

Test the effects of anti-cancer drugs such as peptidomimetic ligands (PBP1 and PBP2) targeting G4s (daunomycin, PBP2) and IMs (PBP1) on LTP and LTD ${ }^{27,93}$.

Test the effects of de-methylation/methylation drugs (especially targeting DNMT3A) on $\mathrm{IM} /$ triplex formation and $\mathrm{G} 4$ dynamics and stability in neurons by utilizing in vitro nuclear magnetic resonance (NMR) spectroscopy (methods mentioned above).

$\underline{\text { In vivo: }}$

Detect IM and G4 and monitor their dynamics in the process of new memory formation (e.g. fear conditioning/habitual avoidance and after its extinction or drug treatment) using specific anti-IM or anti-G4 antibodies ${ }^{6,88}$.

In the human genome, it has recently been demonstrated that G4s are associated with CGI hypomethylation where they inhibit DNMT1 enzymatic activity and thereby protect certain CGls from methylation ${ }^{15}$.

Detect in vivo DNA methylation in putative IM/G4 in genes (including regulatory regions) involved in neuroplasticity (ideally at different stages of LTP/LTD, etc). It would be ideal to determine specific methylation sites and whether it is $\mathrm{CpG}$ or non-CpG methylation. Further 
investigation into disorder specific IM/G4 formation and DNA methylation may help develop targeted treatments with lower side effects.

Investigate the proposed use of qPCR to detect methylation via effect on IM and $\mathrm{G} 4^{13}$.

Test the effects of IM and/G4 disrupting drugs such as peptidomimetic ligands (PBP1 and PBP2) targeting G4s (daunomycin, PBP2) and IMs (PBP1) on neuroplasticity involved in metal health disorders such as ASD and OCD mice models ${ }^{27,93}$.

Find physiologically active miRNAs likely to bind to IM, G4 and triplex forming sites (from literature and by homology search). Test their effects with or without drugs on the stability on these non-canonical structures in vivo ${ }^{27}$.

\section{Conclusions}

In summary, we hypothesize that non-canonical DNA and RNA structures are an integral part of neuroplasticity control via gene expression regulation at the level of transcription, translation and splicing. We propose that the regulatory activity of DNA IM and G4 structures is modulated by DNA methylation/demethylation of the IM and/or G4 sequences, which facilitates the switch between canonical and non-canonical conformation. Other neuronal mechanisms interacting with the formation and regulatory activity of non-canonical DNA and RNA structures, particularly G4, IM and triplexes, may involve microRNAs as well as ion and proton fluxes. The studies we propose could yield new insights into the essential neuronal mechanisms involved in memory and behavior while possibly generating novel approaches for drug and treatment development for a broad spectrum of mental and neurological illnesses.

\section{Competing interests statement}

All author declare that they have no significant competing financial, professional, or personal interests that might have influenced the performance or presentation of the work described in this manuscript. 


\section{References}

1 Abou Assi, H., Garavis, M., Gonzalez, C. \& Damha, M. J. i-Motif DNA: structural features and significance to cell biology. Nucleic Acids Res 46, 8038-8056, doi:10.1093/nar/gky735 (2018).

2 Gehring, K., Leroy, J.-L. \& Guéron, M. A tetrameric DNA structure with protonated cytosinecytosine base pairs. Nature 363, 561, doi:10.1038/363561a0 (1993).

3 Sen, D. \& Gilbert, W. Formation of parallel four-stranded complexes by guanine-rich motifs in DNA and its implications for meiosis. Nature 334, 364, doi:10.1038/334364a0 (1988).

4 Benabou, S., Aviñó, A., Eritja, R., González, C. \& Gargallo, R. Fundamental aspects of the nucleic acid i-motif structures. RSC Advances 4, 26956-26980, doi:10.1039/C4RA02129K (2014).

5 Leroy, J. L., Gehring, K., Kettani, A. \& Gueron, M. Acid multimers of oligodeoxycytidine strands: stoichiometry, base-pair characterization, and proton exchange properties. Biochemistry 32, 6019-6031 (1993).

6 Zeraati, M. et al. I-motif DNA structures are formed in the nuclei of human cells. Nat Chem 10, 631-637, doi:10.1038/s41557-018-0046-3 (2018).

7 Armas, P. \& Calcaterra, N. B. G-quadruplex in animal development: Contribution to gene expression and genomic heterogeneity. Mechanisms of Development, doi:https://doi.org/10.1016/j.mod.2018.05.004 (2018).

8 Yoshida, W., Terasaka, M., Laddachote, S. \& Karube, I. Stabilization of G-quadruplex structure on vascular endothelial growth factor gene promoter depends on $\mathrm{CpG}$ methylation site and cation type. Biochimica et Biophysica Acta (BBA) - General Subjects 1862, 1933-1937, doi:https://doi.org/10.1016/j.bbagen.2018.06.014 (2018).

9 Biffi, G., Tannahill, D., McCafferty, J. \& Balasubramanian, S. Quantitative visualization of DNA Gquadruplex structures in human cells. Nat Chem 5, 182-186, doi:10.1038/nchem.1548 (2013). 
10 Brooks, T. A., Kendrick, S. \& Hurley, L. Making sense of G-quadruplex and i-motif functions in oncogene promoters. FEBS J 277, 3459-3469, doi:10.1111/j.1742-4658.2010.07759.x (2010).

11 Smith, I. M. et al. Coordinated activation of candidate proto-oncogenes and cancer testes antigens via promoter demethylation in head and neck cancer and lung cancer. PLoS One 4, e4961, doi:10.1371/journal.pone.0004961 (2009).

12 Tsukakoshi, K., Saito, S., Yoshida, W., Goto, S. \& Ikebukuro, K. CpG Methylation Changes GQuadruplex Structures Derived from Gene Promoters and Interaction with VEGF and SP1. Molecules 23, doi:10.3390/molecules23040944 (2018).

13 Yoshida, W. et al. Detection of DNA Methylation of G-Quadruplex and i-Motif-Forming Sequences by Measuring the Initial Elongation Efficiency of Polymerase Chain Reaction. Analytical Chemistry 88, 7101-7107, doi:10.1021/acs.analchem.6b00982 (2016).

14 Stevens, A. J. \& Kennedy, M. A. Methylated Cytosine Maintains G-Quadruplex Structures during Polymerase Chain Reaction and Contributes to Allelic Dropout. Biochemistry 56, 3691-3698, doi:10.1021/acs.biochem.7b00480 (2017).

15 Mao, S. Q. et al. DNA G-quadruplex structures mold the DNA methylome. Nat Struct Mol Biol 25, 951-957, doi:10.1038/s41594-018-0131-8 (2018).

16 Xu, B., Devi, G. \& Shao, F. Regulation of telomeric i-motif stability by 5-methylcytosine and 5hydroxymethylcytosine modification. Org Biomol Chem 13, 5646-5651, doi:10.1039/c4ob02646b (2015).

17 Isaakova E, V. A. a. P. G. Cpg Methylation in G-Quadruplex and IMotif

DNA Structures. Crimson Publishers (2018).

18 Miikkulainen, R. \& Moll, M. Convergence-Zone Episodic Memory: Analysis and Simulations. Neural Netw 10, 1017-1036 (1997). 
19 Moriarty, D. E. \& Miikkulainen, R. Forming neural networks through efficient and adaptive coevolution. Evol Comput 5, 373-399 (1997).

20 Miikkulainen, R., Bednar, J. A., Choe, Y. \& Sirosh, J. in Psychology of Learning and Motivation Vol. 36 (eds Robert L. Goldstone, Douglas L. Medin, \& Philippe G. Schyns) 257-308 (Academic Press, 1997).

21 Sirosh, J. \& Miikkulainen, R. Topographic receptive fields and patterned lateral interaction in a self-organizing model of the primary visual cortex. Neural Comput 9, 577-594 (1997).

22 Sierra, A., Encinas, J. M. \& Maletic-Savatic, M. Adult human neurogenesis: from microscopy to magnetic resonance imaging. Front Neurosci 5, 47, doi:10.3389/fnins.2011.00047 (2011).

23 Jeanneteau, F. \& Arango-Lievano, M. Linking Mitochondria to Synapses: New Insights for StressRelated Neuropsychiatric Disorders. Neural Plast 2016, 3985063, doi:10.1155/2016/3985063 (2016).

24 Nikoletopoulou, V. \& Tavernarakis, N. Regulation and Roles of Autophagy at Synapses. Trends in Cell Biology 28, 646-661, doi:https://doi.org/10.1016/j.tcb.2018.03.006 (2018).

25 Kendrick, S., Akiyama, Y., Hecht, S. M. \& Hurley, L. H. The i-motif in the bcl-2 P1 promoter forms an unexpectedly stable structure with a unique 8:5:7 loop folding pattern. Journal of the American Chemical Society 131, 17667-17676, doi:10.1021/ja9076292 (2009).

26 Le, V. H., Nagesh, N. \& Lewis, E. A. Bcl-2 promoter sequence G-quadruplex interactions with three planar and non-planar cationic porphyrins: TMPyP4, TMPyP3, and TMPyP2. PloS one 8, e72462-e72462, doi:10.1371/journal.pone.0072462 (2013).

27 Debnath, M. et al. Preferential targeting of i-motifs and G-quadruplexes by small molecules. Chemical science 8, 7448-7456, doi:10.1039/c7sc02693e (2017).

28 Hoeffer, C. A. \& Klann, E. mTOR signaling: at the crossroads of plasticity, memory and disease. Trends in neurosciences 33, 67-75, doi:10.1016/j.tins.2009.11.003 (2010). 
29 Graber, T. E., McCamphill, P. K. \& Sossin, W. S. A recollection of mTOR signaling in learning and memory. Learn Mem 20, 518-530, doi:10.1101/Im.027664.112 (2013).

30 Takei, N. \& Nawa, H. mTOR signaling and its roles in normal and abnormal brain development. Frontiers in Molecular Neuroscience 7, doi:10.3389/fnmol.2014.00028 (2014).

31 Haeusler, A. R. et al. C9orf72 nucleotide repeat structures initiate molecular cascades of disease. Nature 507, 195-200, doi:10.1038/nature13124 (2014).

32 Kunugi, H. et al. Association study of structural mutations of the tyrosine hydroxylase gene with schizophrenia and Parkinson's disease. Am J Med Genet 81, 131-133 (1998).

33 Bademci, G., Vance, J. M. \& Wang, L. Tyrosine hydroxylase gene: another piece of the genetic puzzle of Parkinson's disease. CNS \& neurological disorders drug targets 11, 469-481 (2012).

34 Banerjee, K. et al. Regulation of tyrosine hydroxylase transcription by hnRNP K and DNA secondary structure. Nature Communications 5, 5769, doi:10.1038/ncomms6769

https://www.nature.com/articles/ncomms6769\#supplementary-information (2014).

35 Farhath, M. M. et al. G-Quadruplex-Enabling Sequence within the Human Tyrosine Hydroxylase Promoter Differentially Regulates Transcription. Biochemistry 54, 5533-5545, doi:10.1021/acs.biochem.5b00209 (2015).

36 Guan, J.-S., Xie, H. \& Ding, X. The role of epigenetic regulation in learning and memory. Experimental Neurology 268, 30-36, doi:https://doi.org/10.1016/j.expneurol.2014.05.006 (2015).

37 Kundakovic, M. in Medical Epigenetics (ed T. O. Tollefsbol) 335-350 (Academic Press, 2016).

38 Lubin, F. D., Gupta, S., Parrish, R. R., Grissom, N. M. \& Davis, R. L. Epigenetic mechanisms: critical contributors to long-term memory formation. Neuroscientist 17, 616-632, doi:10.1177/1073858411386967 
10.1177/1073858410386967 (2011).

39 Woldemichael, B. T., Bohacek, J., Gapp, K. \& Mansuy, I. M. Epigenetics of memory and plasticity. Prog Mol Biol Transl Sci 122, 305-340, doi:10.1016/B978-0-12-420170-5.00011-8 (2014).

40 Bagga, J. S. \& D'Antonio, L. A. Role of conserved cis-regulatory elements in the posttranscriptional regulation of the human MECP2 gene involved in autism. Hum Genomics 7, 19, doi:10.1186/1479-7364-7-19 (2013).

41 Jang, H. S., Shin, W. J., Lee, J. E. \& Do, J. T. CpG and Non-CpG Methylation in Epigenetic Gene Regulation and Brain Function. Genes (Basel) 8, doi:10.3390/genes8060148 (2017).

42 Morris, M. J., Na, E. S., Autry, A. E. \& Monteggia, L. M. Impact of DNMT1 and DNMT3a forebrain knockout on depressive- and anxiety like behavior in mice. Neurobiology of learning and memory 135, 139-145, doi:10.1016/j.nIm.2016.08.012 (2016).

43 Kozlenkov, A. et al. A unique role for DNA (hydroxy)methylation in epigenetic regulation of human inhibitory neurons. Sci Adv 4, eaau6190, doi:10.1126/sciadv.aau6190 (2018).

44 Shioda, N. et al. Targeting G-quadruplex DNA as cognitive function therapy for ATR-X syndrome. Nat Med 24, 802-813, doi:10.1038/s41591-018-0018-6 (2018).

45 Xu, B., Hsu, P.-K., Karayiorgou, M. \& Gogos, J. A. MicroRNA dysregulation in neuropsychiatric disorders and cognitive dysfunction. Neurobiology of disease 46, 291-301, doi:10.1016/j.nbd.2012.02.016 (2012).

46 Rouleau, S., Glouzon, J. S., Brumwell, A., Bisaillon, M. \& Perreault, J. P. 3' UTR G-quadruplexes regulate miRNA binding. RNA 23, 1172-1179, doi:10.1261/rna.060962.117 (2017).

47 Rouleau, S. G., Garant, J.-M., Bolduc, F., Bisaillon, M. \& Perreault, J.-P. G-Quadruplexes influence pri-microRNA processing. RNA Biology 15, 198-206, doi:10.1080/15476286.2017.1405211 (2018). 
48 Pandey, S., Agarwala, P., Jayaraj, G. G., Gargallo, R. \& Maiti, S. The RNA Stem-Loop to GQuadruplex Equilibrium Controls Mature MicroRNA Production inside the Cell. Biochemistry 54, 7067-7078, doi:10.1021/acs.biochem.5b00574 (2015).

49 Costa-Mattioli, M., Sossin, W. S., Klann, E. \& Sonenberg, N. Translational control of long-lasting synaptic plasticity and memory. Neuron 61, 10-26, doi:10.1016/j.neuron.2008.10.055 (2009).

50 Rosenberg, T. et al. The roles of protein expression in synaptic plasticity and memory consolidation. Frontiers in molecular neuroscience 7, 86-86, doi:10.3389/fnmol.2014.00086 (2014).

51 Uttam, S., Wong, C., Price, T. J. \& Khoutorsky, A. elF4E-Dependent Translational Control: A Central Mechanism for Regulation of Pain Plasticity. Frontiers in genetics 9, 470-470, doi:10.3389/fgene.2018.00470 (2018).

52 Ainsley, J. A., Drane, L., Jacobs, J., Kittelberger, K. A. \& Reijmers, L. G. Functionally diverse dendritic mRNAs rapidly associate with ribosomes following a novel experience. Nature Communications 5, 4510, doi:10.1038/ncomms5510

https://www.nature.com/articles/ncomms5510\#supplementary-information (2014).

53 Sutton, M. A. \& Schuman, E. M. Dendritic protein synthesis, synaptic plasticity, and memory. Cell 127, 49-58, doi:10.1016/j.cell.2006.09.014 (2006).

54 Ostroff, L. E., Fiala, J. C., Allwardt, B. \& Harris, K. M. Polyribosomes redistribute from dendritic shafts into spines with enlarged synapses during LTP in developing rat hippocampal slices. Neuron 35, 535-545 (2002).

55 Bugaut, A. \& Balasubramanian, S. 5'-UTR RNA G-quadruplexes: translation regulation and targeting. Nucleic acids research 40, 4727-4741, doi:10.1093/nar/gks068 (2012).

56 Guo, J. U. \& Bartel, D. P. RNA G-quadruplexes are globally unfolded in eukaryotic cells and depleted in bacteria. Science 353, doi:10.1126/science.aaf5371 (2016). 
57 Song, J., Perreault, J.-P., Topisirovic, I. \& Richard, S. RNA G-quadruplexes and their potential regulatory roles in translation. Translation (Austin, Tex.) 4, e1244031-e1244031, doi:10.1080/21690731.2016.1244031 (2016).

58 Fay, M. M., Lyons, S. M. \& Ivanov, P. RNA G-Quadruplexes in Biology: Principles and Molecular Mechanisms. J Mol Biol 429, 2127-2147, doi:10.1016/j.jmb.2017.05.017 (2017).

59 Huang, H., Zhang, J., Harvey, S. E., Hu, X. \& Cheng, C. RNA G-quadruplex secondary structure promotes alternative splicing via the RNA-binding protein hnRNPF. Genes \& development 31, 2296-2309, doi:10.1101/gad.305862.117 (2017).

60 Kwok, C. K., Marsico, G. \& Balasubramanian, S. Detecting RNA G-Quadruplexes (rG4s) in the Transcriptome. Cold Spring Harbor perspectives in biology 10, doi:10.1101/cshperspect.a032284 (2018).

61 Caputi, M. \& Zahler, A. M. Determination of the RNA binding specificity of the heterogeneous nuclear ribonucleoprotein (hnRNP) H/H'/F/2H9 family. J Biol Chem 276, 43850-43859, doi:10.1074/jbc.M102861200 (2001).

62 Cammas, A. et al. hnRNP A1-mediated translational regulation of the G quadruplex-containing RON receptor tyrosine kinase mRNA linked to tumor progression. Oncotarget 7, 16793-16805, doi:10.18632/oncotarget.7589 (2016).

63 Simone, R., Fratta, P., Neidle, S., Parkinson, G. N. \& Isaacs, A. M. G-quadruplexes: Emerging roles in neurodegenerative diseases and the non-coding transcriptome. FEBS Letters 589, 1653-1668, doi:https://doi.org/10.1016/j.febslet.2015.05.003 (2015).

64 Cammas, A. \& Millevoi, S. RNA G-quadruplexes: emerging mechanisms in disease. Nucleic Acids Res 45, 1584-1595, doi:10.1093/nar/gkw1280 (2017). 
65 Blice-Baum, A. C. \& Mihailescu, M. R. Biophysical characterization of G-quadruplex forming FMR1 mRNA and of its interactions with different fragile $X$ mental retardation protein isoforms. RNA 20, 103-114, doi:10.1261/rna.041442.113 (2014).

66 Blackwell, E., Zhang, X. \& Ceman, S. Arginines of the RGG box regulate FMRP association with polyribosomes and mRNA. Hum Mol Genet 19, 1314-1323, doi:10.1093/hmg/ddq007 (2010).

67 Zhang, Y., Gaetano, C. M., Williams, K. R., Bassell, G. J. \& Mihailescu, M. R. FMRP interacts with G-quadruplex structures in the 3'-UTR of its dendritic target Shank1 mRNA. RNA Biol 11, 13641374, doi:10.1080/15476286.2014.996464 (2014).

68 McAninch, D. S. et al. Fragile X mental retardation protein recognizes a $\mathrm{G}$ quadruplex structure within the survival motor neuron domain containing 1 mRNA 5'-UTR. Molecular bioSystems 13, 1448-1457, doi:10.1039/c7mb00070g (2017).

69 Nguyen, T., Fraire, C. \& Sheardy, R. D. Linking pH, Temperature, and K(+) Concentration for DNA i-Motif Formation. J Phys Chem B 121, 7872-7877, doi:10.1021/acs.jpcb.7b06317 (2017).

70 Saxena, S., Joshi, S., Shankaraswamy, J., Tyagi, S. \& Kukreti, S. Magnesium and molecular crowding of the cosolutes stabilize the i-motif structure at physiological pH. Biopolymers 107, doi:10.1002/bip.23018 (2017).

71 Bhattacharyya, D., Mirihana Arachchilage, G. \& Basu, S. Metal Cations in G-Quadruplex Folding and Stability. Frontiers in chemistry 4, 38-38, doi:10.3389/fchem.2016.00038 (2016).

72 Lin, C.-T., Tseng, T.-Y. \& Chang*, T.-C. Structural Diversity of G-Quadruplexes: Potassium Concentration Effect. Biophysical Journal 98, 265a, doi:10.1016/j.bpj.2009.12.1442 (2010).

73 Svinarchuk, F., Cherny, D., Debin, A., Delain, E. \& Malvy, C. A new approach to overcome potassium-mediated inhibition of triplex formation. Nucleic acids research $\mathbf{2 4}, \mathbf{3 8 5 8 - 3 8 6 5}$ (1996). 
74 Tan, Z.-J. \& Chen, S.-J. RNA helix stability in mixed Na+/Mg2+ solution. Biophysical journal 92, 3615-3632, doi:10.1529/biophysj.106.100388 (2007).

75 Wanrooij, P. H. et al. A hybrid G-quadruplex structure formed between RNA and DNA explains the extraordinary stability of the mitochondrial R-loop. Nucleic acids research 40, 10334-10344, doi:10.1093/nar/gks802 (2012).

76 Lacroix, L., Mergny, J.-L., Leroy, J.-L. \& Hélène, C. Inability of RNA To Form the i-Motif: Implications for Triplex Formation. Biochemistry 35, 8715-8722, doi:10.1021/bi960107s (1996).

77 Chesler, M. Regulation and Modulation of pH in the Brain. Physiological Reviews 83, 1183-1221, doi:10.1152/physrev.00010.2003 (2003).

78 Brooks, K. J. \& Bachelard, H. S. The regulation of intracellular pH studied by 31P-and $1 \mathrm{H}-\mathrm{NMR}$ spectroscopy in superfused guinea-pig cerebral cortex slices. Neurochem Int 21, 375-379 (1992).

79 Diering, G. \& Numata, M. Endosomal pH in neuronal signaling and synaptic transmission: role of $\mathrm{Na}+/ \mathrm{H}+$ exchanger NHE5. Frontiers in Physiology 4, doi:10.3389/fphys.2013.00412 (2014).

80 Breckwoldt, M. O. et al. Mitochondrial redox and pH signaling occurs in axonal and synaptic organelle clusters. Scientific Reports 6, 23251, doi:10.1038/srep23251

https://www.nature.com/articles/srep23251\#supplementary-information (2016).

81 Sinning, A. \& Hubner, C. A. Minireview: pH and synaptic transmission. FEBS Lett 587, 1923-1928, doi:10.1016/j.febslet.2013.04.045 (2013).

82 Choi, J., Kim, S., Tachikawa, T., Fujitsuka, M. \& Majima, T. pH-induced intramolecular folding dynamics of i-motif DNA. J Am Chem Soc 133, 16146-16153, doi:10.1021/ja2061984 (2011).

83 Goni, J. R., de la Cruz, X. \& Orozco, M. Triplex-forming oligonucleotide target sequences in the human genome. Nucleic Acids Res 32, 354-360, doi:10.1093/nar/gkh188 (2004).

84 Kendrick, S. et al. The Dynamic Character of the BCL2 Promoter i-Motif Provides a Mechanism for Modulation of Gene Expression by Compounds That Bind Selectively to the Alternative DNA 
Hairpin Structure. Journal of the American Chemical Society 136, 4161-4171, doi:10.1021/ja410934b (2014).

85 Amato, J., laccarino, N., Randazzo, A., Novellino, E. \& Pagano, B. Noncanonical DNA Secondary Structures as Drug Targets: the Prospect of the i-Motif. ChemMedChem 9, 2026-2030, doi:10.1002/cmdc.201402153 (2014).

86 Buket, O., Clement, L. \& DanZhou, Y. DNA G-quadruplex and its potential as anticancer drug target. Science China. Chemistry 57, 1605-1614, doi:10.1007/s11426-014-5235-3 (2014).

87 Asamitsu, S., Obata, S., Yu, Z., Bando, T. \& Sugiyama, H. Recent Progress of Targeted GQuadruplex-Preferred Ligands Toward Cancer Therapy. Molecules 24, 429 (2019).

88 Henderson, A. et al. Detection of G-quadruplex DNA in mammalian cells. Nucleic Acids Res 42, 860-869, doi:10.1093/nar/gkt957 (2014).

89 Mergny, J. L. et al. Telomerase inhibitors based on quadruplex ligands selected by a fluorescence assay. Proc Natl Acad Sci U S A 98, 3062-3067, doi:10.1073/pnas.051620698 (2001).

90 Gasparovic, C., King, D. \& Feeney, D. M. Metabolism in single rat brain slices measured by magnetic resonance spectroscopy. Brain research. Brain research protocols 4, 97-102 (1999).

91 Litt, L., Hirai, K., Basus, V. J. \& James, T. L. Temperature control of respiring rat brain slices during high field NMR spectroscopy. Brain Research Protocols 10, 191-198, doi:https://doi.org/10.1016/S1385-299X(02)00218-0 (2003).

92 Espanol, M. T. et al. Adult rat brain-slice preparation for nuclear magnetic resonance spectroscopy studies of hypoxia. Anesthesiology 84, 201-210 (1996).

93 Barthwal, R. \& Tariq, Z. Molecular Recognition of Parallel G-quadruplex [d-(TTGGGGT) $]_{4}$ Containing Tetrahymena Telomeric DNA Sequence by Anticancer Drug Daunomycin: NMR-Based Structure and Thermal Stability. Molecules (Basel, Switzerland) 23, 2266, doi:10.3390/molecules23092266 (2018). 


\section{Additional literature}

Special Journal Issue on IM/G4: https://www.mdpi.com/journal/molecules/special_issues/Gquadruplexes_i-motif_DNA 